\title{
Assessment of 3 Lateral Pins Fixation in the Management of the Supracondylar Humeral Fractures in Children
}

\author{
M.A.Mashhour, S.A.Al Traigy and A.E.El-Sheikh \\ Orthopedic Surgery Dept., Faculty of Medicine, Benha Univ., Benha, Egypt \\ E-Mail: dr_magico@yahoo.com.
}

\begin{abstract}
Supracondylar fractures of the humerus are the most common type of elbow fracture in children, accounting more than $80 \%$ of all fractures. Posterior Displacement suggests a hyper extension injury, normally fall on out extended hand. While foremost removal, uncommon sort, as a rule because of direct viciousness with the joint in flexion. Assessment of the procedure of 3 horizontal pins obsession as a line of treatment of the supracondylar humeral cracks in 20 patients with uncommon spotlight on resultant steadiness, results and entanglements experienced during the investigation time frame. an imminent, randomized controlled clinical preliminary from September 2018 to December 2019. The convention was endorsed by the moral council of the staff of medication, Benha University. Educated assent was taken, from guardians, all things considered, that the information of the examination will be distributed. 13 patients were female and 7 were male. 13 were correct sided and 7 were left sided.The mean age of the patients was 6.05 with range from 3 to 11. Patients were followed up for a very long time normal, and were evaluated by Flynn's models for reviewing and the endproduct were: 15 patients had amazing outcomes (75\%) and 5 had great outcomes (25\%).As for expected intricacies, we noticed no iatrogenic ulnar nerve injury. mean of usable time was 50.25 with range from 35 to 60 ; mean of chunk length was 3.40 with range from 3 to about a month So, employable time is huge here as it required some investment than exemplary obsession either with 2 sidelong pins or 2 parallel pins with 1 average on account of the higher specialized requests of this example of obsession, likewise it required some investment to accomplish strength of fracture.Slab span and expulsion of pins here are critical, as we can eliminate section and pins in normal 3.40 weeks which is quicker than some other example of obsession of SCHF in youngsters . treatment of the supracondylar humeral breaks in kids by shut decrease and interior obsession with 3 sidelong $\mathrm{k}$ wires whenever set with propped procedure is steady and viable strategy for obsession with completley ulnar nerve insurance so it gives great practical and cosmotic result .
\end{abstract}

Keywords: Fracture, Supracondylar humerus, Closed reduction, Lateral 3 wires.

\section{Introduction}

Supracondylar humerus cracks (SCHF) are normal pediatric wounds, speaking to around $4-6.5 \%$ of every pediatric break, they are considered as the most regular cracks in youngsters under 8 years old, and the most wellknown elbow breaks represents $60 \%$ of all cracks around the elbow. Young men have a higher frequency of this sort of fracture [1].

The augmentation sort of SCHF is more than the flexion type which is unprecedented representing just 2$10 \%$ of all SCFH [2].

Gartland arranged supracondylar humeral cracks in kids into three kinds: Type I undisplaced break, type II uprooted break with unblemished back cortex, and type III dislodged crack with no cortical contact [3].

The writing underpins shut decrease and percutaneous sticking as the treatment of decision for these cracks. In any case, this could be related with different confusions, for example, neurovascular bargain going from 5-30\%, skin issues, compartment disorder, Volkmann's ischemia, and cubitus varus with a frequency as high as $60 \%$ [4].

Shut decrease and percutaneous sticking is grounded as the favored careful intercession for uprooted supracondylar crack humerus in children [5]. While accessible information reliably recommend that steady obsession of the break can be accomplished utilizing a few K-wires, the ideal pin arrangement is as yet dubious. Alternatives incorporate average and horizontal crossed pins, two sidelong pins joined with one average pin or parallel pins as it were. Horizontal pins might be embedded in a different, equal or united manner [6]. Current course readings and studies portray the crossed-pin system as the ideal treatment, aside from when the average epicondyle or the ulnar nerve can't be palpated [7,8,9].

Nonetheless, a few investigations report that percutaneous sidelong sticking whenever performed accurately, is viable in keeping up decrease and soundness of supracondylar crack humerus in children $[8,9,10]$.

\section{Aim of the Work}

The point of this examination is to assess the strategy of 3 parallel pins obsession as a line of treatment of the supracondylar humeral breaks in 20 patients with uncommon spotlight on resultant security, results and inconveniences experienced during the investigation time frame.

\section{Patients and methods}

A prospective study included twenty patients at Benha University Hospital, Kafr Elsheikh General Hospital and El Mahalla General Hospital Starting from September 2018 to December 2019 .

\section{Inclusion criteria}

1- Patients with supracondylar humeral crack

2- Age $<12$ years.

3- Closed wounds .

4- Complete $\mathrm{x}$-beam documentation .

5- Accurate radiological documentation previously or after the medical procedure.

6- Patient introducing after under multi week . 


\section{Avoidance criteria}

Any cases with the accompanying models will be prohibited:

1- Incomplete X-beam documentation.

2- Inaccurate radiological documentation previously or after the medical procedure.

3- Age $>12$ years

4- Open wounds .

5- Patient introducing after over multi week

\section{Patients}

$35 \%$ of patients were male yet $65 \%$ of them were female, mean old enough was 6.05 with range from 3 to 11 .

\section{Methods}

The clinical information of the patients satisfying the consideration measures was assessed as follows:

\section{1- Clinical Data}

All patients were assessed clinically by a set of experiences and actual assessment.

The patients were followed clinically for:

- Criteria of Flynn: scope of development and conveying point.

- Neurological and vascular evaluation.

- Infection

\section{2- Radiographic assessment}

- Antero-back view (Baumann point, metaphysealdiaphyseal point)

- lateral view (humero-capitellar point)

\section{Employable procedure}

At first, the patient was analyzed and fitting X-beams had been finished. The upper appendage was supported with the elbow in an agreeable situation of around 20 to 40 levels of flexion, while abstaining from tight gauzing or bracing. The elbow and hand were then delicately raised over the heart. The neuro-vascular assessment was done and recorded.

\section{Sedation}

Medical procedure was done under broad sedation and on enlistment, the patient got intravenous anti-toxin; whose portion was changed by the weight.

\section{Position}

All the patients were situated prostrate with the broke elbow on the wide finish of the fluoroscopy unit as the table. In instances of bizarre insecurity in which pivot of the arm took a chance with the deficiency of decrease, the child ${ }^{\text {ee }}$ s shoulder was situated to be adaptable enough to consider 90 levels of outside revolution to securely acquire a horizontal of the elbow. Additionally, the child $\mathrm{s}$ arm was far enough onto wide finish of the fluoroscopy unit that the elbow was very much pictured with fluoroscopy. At that point the patientee $\mathrm{s}$ arm was then hung and prepared.

\section{Decrease}

At that point shut decrease was performed under the fluoroscopic direction. To begin with, footing was applied with the elbow flexed at around 20 degrees to maintain a strategic distance from the chance of tying neurovascular structures over an anteriorly dislodged proximal section, with the specialist getting a handle on the lower arm with two hands, and the aide giving counter-foothold in the axilla, Next, with the elbow practically straight, varus and valgus rakish arrangement was revised by development of the lower arm. Likewise average and horizontal break interpretation was realigned with direct development of the distal section by the specialist with picture affirmation ,The elbow was then gradually flexed while applying front strain to the olecranon with the surgeon "s thumb, and put in pronation on account of posterio-medially uprooted crack and in supination on account of posterio-along the side dislodged crack.

\section{Following a fruitful decrease}

The child ${ }^{\text {ee }}$ elbow adequately flexed with the goal that the fingers contact the shoulder, the decrease was checked by fluoroscopic pictures in AP, sidelong, and slanted planes, three focuses were check for a decent decrease: (a) the AHL crosses the capitellum,(b)the average and horizontal segments are unblemished on diagonal perspectives.

\section{Sticking method}

When decrease was agreeable, the associate held the elbow in the diminished situation of elbow hyper flexion to forestall loss of decrease while sticking. the main K-wire is set against the horizontal condyle without puncturing skin and checked under antero-back fluoroscopic direction to guarantee the capitellar beginning stage (the focal point of sidelong condyle). For greatest control push the wire through the skin and into the ligament prior to turning on the drill. The pin is pointed $35^{\circ}$ upwards and $10^{\circ}$ back, ought to maintain a strategic distance from the olecranon fossa and should simply penetrate the far cortex. As the focal point of capitellum is in accordance with foremost part of humeral shaft, the wire is embedded through the capitellum and afterward the distal humeral physis.

The point of pin situation is to maximally isolate the pins at the crack site to draw in both the average and parallel sections. A third pin is added to improve steadiness, or if the initial 2 pins are not adequately isolated to connect with both the average and horizontal segment.

After pin arrangement, the decrease is again checked under fluoroscopy with antero-back, parallel, and diagonal perspectives. Likewise, conveying point and Bauman's point are surveyed. At last the outspread heartbeat and the nature of the beat reevaluated, pin ought to cross $1-2 \mathrm{~cm}$ proximal to the crack at a point of about $30^{\circ}$ to the humeral shaft. The wires were then twisted and cut external the skin. Elbow was immobilized with back section with elbow in 70 to 90 level of flexion relying on the expanding and neurovascular status. All patients were given twofold 
portion of wide range anti-infection agents followed by oral anti-toxins 1 week.

\section{Follow up}

All youngsters should be seen week after week for about fourteen days, at that point like clockwork as long as about a month and a half. Piece should be taken out 3 to about a month with $\mathrm{x}$-beam assessment to guarantee solidness.

- Pins should be ceased 3 a month postoperatively when radiological mending is seen .
- Range of movement practices are started soon after pins , immobilization are ended and should be delicate detached and dynamic helped .

- Return to full action commonly happens by 6 to about two months postoperatively

- The persistent and the guardians are cautioned against incredible activities.

\section{Results}

In this study, 20 patients were followed up for 9 months average, and were assessed according to Flynn's criteria for grading and the final results were: 15 patients had excellent results (75\%) and 5 had good results (25\%).

Age, sex, side ,mode of injury and Gartland gading of fracture were insignificant in my study.

\begin{tabular}{lc}
\hline Age (years) & Non significant \\
Sex & Non significant \\
Side & Non significant \\
Mode of injury & Non significant \\
Gartland classification & Non significant \\
Time to Surgery & Non significant \\
Operative Time "Minutes" & Significant \\
\hline
\end{tabular}

Carrying angle loss and motion loss.

\begin{tabular}{llll}
\hline & & No & $\%$ \\
\hline \multirow{2}{*}{ Carrying angle loss(degree) } & $(5-10)$ & 5 & $25.0 \%$ \\
\multirow{2}{*}{ Motion loss } & $0-5$ & 15 & $75.0 \%$ \\
& $(5-10)$ & 6 & $30.0 \%$ \\
& $0-5$ & 14 & $70.0 \%$ \\
\hline
\end{tabular}

This table shows that carrying angle loss degree of $75 \%$ of patients was from 0 to 5 , motion loss of $30 \%$ of them was from 5 to 10 .
This Table shows that flynn's criteria for grading of $75 \%$ of patients was excellent but of $25 \%$ was good.

\begin{tabular}{lccc}
\hline & & No & $\%$ \\
\hline Flynn's criteria for grading & EXCELLENT & 15 & $75.0 \%$ \\
Neurologic deficit & GOOD & 5 & $25.0 \%$ \\
Loss of reduction & No & 20 & $100.0 \%$ \\
\hline
\end{tabular}

Cubitus varus and pin tract infection.

\begin{tabular}{lccc}
\hline & & No & $\%$ \\
\hline Cubitus varus & No & 20 & $100.0 \%$ \\
Pin tract infection & 1 case & 1 & $5.0 \%$ \\
\hline
\end{tabular}

This table shows that there was one case of pin tract infection that resolved on oral antibiotics and did not

Operative time, time from injury and slab duration require premature wire removal and cubitus varus was negative in all patients.

\begin{tabular}{ccccc}
\hline Mini & Max & Mean & SD \\
\hline 35 & 60 & 50.25 & 7.34 \\
6 & 24 & 11.40 & 4.71 \\
3 & 4 & 3.40 & 0.50 \\
3 & 4 & 3.40 & 0.50 \\
\hline
\end{tabular}


This table shows that mean of operative time was 50.25 with range from 35 to 60 ; mean of slab duration was 3.40 with range from 3 to 4 .

So, operative time is significant here as it took more time than classic fixation either with 2 lateral pins or 2 lateral pins with 1 medial because of the higher technical demands of this pattern of fixation, also it took more time to achieve stability of fracture.

Slab duration and removal of pins here are significant, as we can remove slab and pins in average 3.40 weeks which is faster than any other pattern of fixation of SCHF in children .

\section{Discussion}

The treatment of supracondylar cracks intends to reestablish anatomical or close to anatomical decrease, early reestablishing elbow work with great ROM, stay away from complexities like neurovascular, deformation, elbow firmness... and so on Diminishing physical and mental effect of the crack on the kids and their folks [11].

Albeit shut decrease and percutaneous K-wire sticking is the as of now acknowledged treatment of dislodged supracondylar cracks of the humerus in kids, there is still contention on the ideal setup of these K-wires with respect to the break strength and ulnar nerve wellbeing [12].

Open decrease has numerous drawbacks. It delays the hospitalization time, has danger of disease and furthermore respects limitation of the elbow movement because of the delicate tissue scars of the careful intervention [13]. CRPP is favored as a flow treatment methodology which stays away from these problems [14]. However, iatrogenic ulnar nerve injury and loss of decreases are the two significant entanglements related with this method [15].

A biomechanical examination of all pin designs were performed by Lee et al. [16,17] in expansion, varus,valgus, inside pivot and outside revolution utilizing a pediatric manufactured bone model. Dissimilar arrangement along the side to forestall ulnar nerve paralysis had enough soundness yet in hub pivot testing, this kind of design had less strength than different setups. In this investigation unique pins gave more dependability than crossed pin in augmentation, and varus testing.

Skaggs et al. [18] found no ulnar nevre paralysis and no decrease was lost in 124 kids dealt with just sidelong section pins. In an other investigation of Skaggs et al. [19] of 141 youngsters who had Gartland type-II crack, 74 were treated with sidelong pins just and 67 were treated with crossed pins. Of 204 kids who had a Gartland type-III crack, 51 were treated with sidelong pins just and 153 were treated with crossed pins. The arrangement of the pins didn't impact the Baumann's point in both Gartland type-II and Gartland type-III cracks.

Three late meta-investigations have analyzed the issue of pin design and iatrogenic nerve injury. In 2010, Slobogean et al. written about 32 preliminaries with 2,639 patients and discovered there was an iatrogenic ulnar nerve injury for each 28 patients treated with crossed pins contrasted with horizontal sticking. In the exact year, Babal et al. written about an orderly survey of 35 articles examining average and sidelong sticking versus horizontal section sticking and found that iatrogenic ulnar nerve injury happened in 40 of 1,171 (3.4\%) of cross-pins and 5 of $738(0.7 \%)$ of parallel passage pins. The 2012

report by Woratanarat et al. included 18 investigations and 1,615 $\mathrm{SCH}$ cracks. They announced the danger of iatrogenic ulnar nerve injury to be 4.3 occasions higher in cross-sticking contrasted with sidelong sticking. They found no distinction in loss of obsession, late deformation, or Flynn rules between the two sorts of pinning [20,21].

The second issue with pin setup is solidness of pin design. Biomechanical investigations of solidness of different pin setups have been fairly deceptive. Two investigations assessed the torsional strength of pin setups and discovered crossed pins to be more grounded than two parallel pins. Shockingly, in these examinations, the two sidelong pins were put promptly contiguous one another and not isolated at the break site as is suggested clinically for horizontal section pins. In engineered humeri study, Srikumaran et al. discovered cross-pins to be more grounded than two sidelong passage pins, yet didn't test three parallel section pins. Lee et al. discovered that two disparate parallel pins isolated at the break site were better than crossed pins in augmentation stacking and varus yet were identical in valgus The more prominent strength seen with difference of the pins was credited to the area of the convergence of the two pins and more noteworthy uniqueness between the two pins, which would consider some buy in the average segment just as the sidelong column [17,18,22].

Kallio et al.(23)advocated that the pins should be pointed toward the back cortex at a point of $10^{\circ}$ with the diaphyseal hub. Unique consideration should be coordinated for ideal pin situation with the horizontal strategies. The utilization of a third pin requires the more average pin to enter the joint and accordingly expands the danger of joint infiltration and disease. It is recommended that the most proper path was to situate the disparate pins on the horizontal cortex.

Sudeep et al. [24] revealed a planned investigation of 66 kids to assess the distinction between obsession by conventional average parallel sticking and customary sidelong sticking. 6 were lost for catch up with mean time of follow up a half year. No significant loss of decrease was seen in both the gatherings where as there was no huge distinction change in Baumann point, change in Humerocapitellar point, Flynn grade, elbow augmentation and flexion, conveying point, absolute scope of movement. This investigation inferred that no critical contrast between the two strategies as respect steadiness however sidelong wires more secure as respect iatrogenic ulnar nerve injury.

Blossom T, Robertson C, Mahar An et al announced that three horizontal section disparate pins were equal to cross-sticking and both were more grounded than two parallel unique pins [25].

Gopinathan NR et al . three horizontal dissimilar pins were comparable to cross pin obsession and both these builds were more grounded than two parallel disparate pins [26].

Lee YH. et al . His examination included 37 sort III cracks and 24 sort II breaks and discovered three 
horizontal pin obsession to be most secure and generally steady as he deduced in his investigation that parallel three disparate Kirschner wires were a protected technique giving sufficient adjustment in Gartland Type III cracks accomplishing fantastic outcomes in $91.8 \%$ patients and great outcomes in $8.2 \%$ patients [27].

Prashant $\mathrm{K}$ discovered brilliant outcomes with horizontal pin obsessions in insecure cracks in this manner dodging iatrogenic nerve injury [28].

Sapkota K and Shrestha B proposed horizontal sticking with 2 or $3 \mathrm{~K}$-wires for legitimate adjustment and ideal design to be disparate to hold average and sidelong sections as the treatment of supracondylar break without danger of iatrogenic ulnar nerve injury [29].

Fellow SP, Ponnuru RR, Gella S, Tulwa N. suggested the utilization of three pins on the sidelong side with no proof of loss of decrease and no iatrogenic nerve injury in their investigation [30].

The creators of an enormous study(19), of 345 patients, reasoned that obsession of both kind II and type-III pediatric SCHFs with just sidelong pins gives satisfactory obsession while evading iatrogenic injury to the ulnar nerve [31].

It has been indicated that three parallel pins give more torsional solidness than do two horizontal pins [32].

The creators closed the significant specialized focuses for obsession with parallel section sticks as follows: [18]

Maximize partition of the pins at the crack site.

Engage the average and horizontal sections proximal to the break

Engage adequate bone in both the proximal section and the distal fragment

Maintain a low limit for utilization of a third horizontal section pin if there is worry about crack steadiness or the area of the initial two pins.

Use three pins for type III fractures.

\section{Summary and conclusion}

Supracondylar breaks of the humerus in youngsters keep on having treatment challenges for the specialist, CRIF through parallel entery versus cross obsession is by all accounts the intriguing issue of discussion.

In this investigation $20 \mathrm{kid}$ experienced supracondylar break of the humerus introduced to Benha University Hospital, kafr El Sheik general clinic and El Mehalla general clinic from September 2018 to October 2019.

All patients were treated by shut decrease and inner obsession with 3 Kirschner wires from horizontal side. The mean age was 7 gone from (3-11) a long time, 13 patients were females (65\%) and 7 patients were guys (35\%),18 patients had their break because of falling on outstretched hand $(90 \%)$ and 2 patients had their cracks because of tumbling from tallness $(10 \%)$, the time from injury to medical procedure range between (6-24) hours, According to Gartland order 18 cases were type 3 (90\%), 2 cases were type 4 (10\%), employable time range was (35-an hour.

As per Flynn's standards , 15 patients had incredible outcomes and 5 patients had great outcomes. Patients were quickly activated after pins removal( 3 to $4 \mathrm{wks})$, the subsequent period was 2 months normal.
In this little investigation we inferred that treatment of the supracondylar humeral cracks in youngsters by shut decrease and inward obsession with 3 parallel $\mathrm{k}$ wires whenever put with propped procedure is steady and successful strategy for obsession with completley ulnar nerve assurance so it gives astounding useful and cosmotic outcome .

\section{References}

[1] HH Noaman, Microsurgical reconstruction of brachial artery injuries in displased supracondylar fracture humerus in children. Microsurgery, Vol.26, PP.498-505. 2006

[2] D.Marshland, S.Belkoff ,Biomechanical analysis of posterior intrafocal pin fixation for the pediatric supracondylar humeral fracture. J Pediatr Orthop, Vol.34(1), PP.40-4,2014.

[3] JJ. Gartland,Management of supracondylar fractures of the humerus in children. Surg Gynecol Obstet, Vol.109, PP.145-154,1959.

[4] T.L.Chen, C.Q.He, T.Q.Zheng, Y.Q.Gan, Stiffness of various pin configurations for pediatric supracondylar humeral fracture: a systematic review on biomechanical studies. J Pediatr Orthop, Vol.24(5), PP.389-99,2015.

[5] A.T.Pennock, C.Michael, M.Molly, T.P.Bastrom ,Potential Causes of Loss of Reduction in Supracondylar Humerus Fractures . J Pediatr Orthop, Vol.34, PP.691-697,2014.

[6] H.N.Ladenhauf, M.Schaffert, J.Bauer,The displaced supracondylar humerus fracture: indications for surgery and surgical options: a 2014 update. Curr Opin Pediatr, Vol.26, PP.64-69,2014.

[7] E.N.Novaisa , P.M.Carryb, B.J.Markc, D.Sayan Posterolaterally displaced and flexion-type supracondylar fractures are associated with a higher risk of open reduction. Journal of Pediatric Orthopaedics B, 2016.

[8] N.E.Green, N.V.Zeeland,Fractures and Dislocations About the Elbow. In Skeletal Trauma in Children, 4th ed, N.E.Green, M.F.Swiontkowski, Vol.Chapter 9, PP.207-282,2009.

[9] W.A.El-Adl, M.A.El-Said, G.W.Boghdady, Results of treatment of displaced supracondylar humeral fractures in children by percutaneous lateral crosswiring technique. Strategies in Trauma and Limb Reconstruction, Springer-Verlag, Vol. 3 (1), PP. 17,2008.

[10] K.Memisoglu, C.C.Kesemenli, H.Atmaca, Does the technique of lateral cross-wiring (Dorgan's technique) reduce iatrogenic ulnar nerve injury? International Orthopaedics (SICOT), PP.35,2011.

[11] C.A.Brauer, B.M.Lee, D.S.Bae, P.M.Waters, systematic review of medial and lateral entry pinning versus lateral entry pinning for supracondylar fractures of the humerus. J Pediatr Orthop, Vol. 2, 27, PP.181-186,2008.

[12] O.Eberhardt, F.Fernandez, T.Ilchmann, K.Parsch, Cross pinning of supracondylar fractures from a 
lateral approach. Stabilization achieved with safety. J Child Orthop, Vol.1, PP.127-133,2007.

[13] A.Aslan, M.N.Konya, A.Ozdemir, H.Yorgancigil, Open reduction and pinning for the treatment of Gartland extension type III supracondylar humeral fractures in children. Strategies in trauma and limb reconstruction., Vol.9(2), PP.79-88,2014.

[14] A.Dua, K.K.Eachempati, R.Malhotra, L.Sharma, Closed reduction and percutaneous pinning of displaced supracondylar fractures of humerus in children with delayed presentation. Chinese journal of traumatology = Zhonghua chuang shang za zhi. , Vol.14(1), PP.14-19,2011.

[15] P.Woratanarat, C. Angsanuntsukh, S .Rattanasiri, J. Attia, Meta-analysis of pinning in supracondylar fracture of the humerus in children. Journal of orthopaedic trauma, Vol.26(1), PP.48-53,2012.

[16] M.Silva, A.R.Knutsen, J.J.Kalma, Biomechanical testing of pin configurations in supracondylar humeral fractures: the effect of medial column comminution. Journal of orthopaedic trauma, Vol.27(5), PP.275-280,2013.

[17] S.S.Lee, A.T.Mahar, P.O.Newton,Displaced pediatric supracondylar humerus fractures: biomechanical analysis of percutaneous pinning techniques. Journal of Pediatric Orthopaedics, Vol.22(4), PP.440-443,2002.

[18] D.L.Skaggs, M.W.Cluck, A.Mostofi, Lateral-entry pin fixation in the management of supracondylar fractures in children. J Bone Joint Surg Am, Vol.86 A (4), PP.702-707,2004.

[19] D.L.Skaggs, J.M.Hale, J.Bassett, C.Kaminsky, Operative treatment of supracondylar fractures of the humerus in children: the consequences of pin placement. JBJS, Vol.83(5), PP.735-740,2001.

[20] J.C.Babal, C.T.Mehlman, G.Klein, Nerve injuries associated with pediatric supracondylar humeral fractures: a meta-analysis. J Pediatr Orthop, Vol.30(3), PP.253-263,2010.

[21] P.Woratanarat, C.Angsanuntsukh, S.Rattanasiri, Meta-analysis of pinning in supracondylar fracture of the humerus in children. $J$ Orthop Trauma, Vol.26(1), PP.48-53,2012.

[22] U.Srikumaran, E.W.Tan, S.M.Belkoff, Enhanced biomechanical stiffness with large pins in the operative treatment of pediatric supracondylar humerus fractures. J Pediatr Orthop, Vol.32(2), PP.201-205.2012.
[23] P.E.Kallio, B.K.Foster, D.C.Paterson,Difficult supracondylar elbow fractures in children: analysis of percutaneous pinning technique. Journal of pediatric orthopedics, Vol.12(1), PP.11-15,1992.

[24] Sudeep Vaidya, Achyut Rajbhandari, Nabees Pradhan, Suman Shrestha,Percutenious Fixation Of Displaced Supracondylar Fracture In Children Comparing Lateral With Medial And Lateral Pin. J Pediatr Orthop, Vol.13, PP.521-555,2009.

[25] T.Bloom, C.Robertson, A.Mahar, M.Pring, Comparison of Supracondylar humeral fracture is not anatomically reduced. In: Read at the Annual Meeting of the Pediatric Orthopaedic society of North America Hollywood, FL,2007.

[26] N.R.Gopinathan, M.Sajid, P.Sudesh and P.Behera, Outcome analysis of lateral pinning for displaced supracondylar fractures in children using three Kirschner Wires in parallel and divergent configuration. Indian journal of orthopaedics, Vol.52(5), PP.554,2018.

[27] Y.H.Lee, S.K.Lee, B.S.Kim, M.S.Chung, Three lateral divergent or parallel pin fixations for the treatment of displaced supracondylar humerus fractures in children. Journal of Pediatric Orthopaedics, Vol. 28(4), PP.417-422,2008.

[28] K.Prashant, A comparative study of two percutaneous pinning techniques (lateral vs medial-lateral) for Gartland type III pediatric supracondylar fracture of the humerus. J Orthop Traumatol, Vol. 17(3), PP.223-229,2016.

[29] K.Sapkota and B.Shrestha, Study of Supracondylar Fracture of Distal Humerus in Children and Its Management with Lateral K-Wire Fixation. Nepal Journal of Medical Sciences, Vol. 3(1), PP.3843,2014.

[30] S.P.Guy, R.R.Ponnuru, S.Gella, N.Tulwa, Lateral entry fixation using three divergent pins for displaced paediatric supracondylar humeral fractures. ISRN Orthop, Vol.2011, PP.137372,2011.

[31] JM. SDaF,Supracondylar Fractures of the Distal Humerus. Rockwood and Wilkins' Fractures in Children; 8th edition, PP.581-624,2015.

[32] 32. M.Silva, A.R.Knutsen, J.J.Kalma, Biomechanical testing of pin configurations in supracondylar humeral fractures: the effect of medial column comminution. Journal of orthopaedic trauma, Vol.27(5), PP.275-280,2013. 